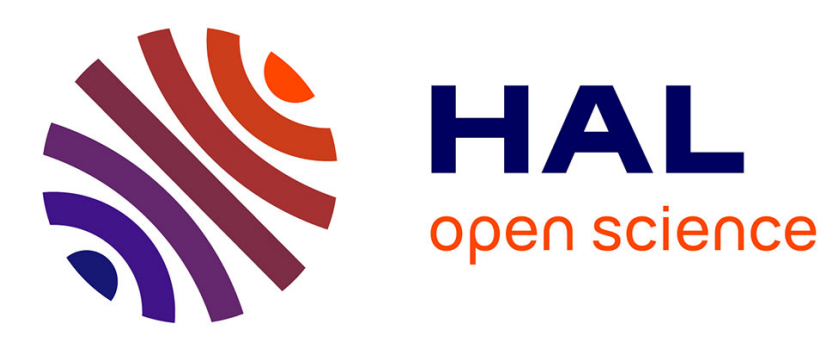

\title{
Robust L2-Gain Observation for structured uncertainties: An LMI approach
}

Benoit Bayon, Gérard Scorletti, Eric Blanco

\section{To cite this version:}

Benoit Bayon, Gérard Scorletti, Eric Blanco. Robust L2-Gain Observation for structured uncertainties: An LMI approach. 50th IEEE CDC - ECC, Dec 2011, Orlando, FL, United States. pp.4949-4954, 10.1109/CDC.2011.6160797 . hal-00655803

\section{HAL Id: hal-00655803 https://hal.science/hal-00655803}

Submitted on 2 Jan 2014

HAL is a multi-disciplinary open access archive for the deposit and dissemination of scientific research documents, whether they are published or not. The documents may come from teaching and research institutions in France or abroad, or from public or private research centers.
L'archive ouverte pluridisciplinaire HAL, est destinée au dépôt et à la diffusion de documents scientifiques de niveau recherche, publiés ou non, émanant des établissements d'enseignement et de recherche français ou étrangers, des laboratoires publics ou privés. 


\title{
Robust $L_{2}$-Gain Observation for structured uncertainties: an LMI approach
}

\author{
Benoit Bayon, Gérard Scorletti, Eric Blanco ${ }^{1}$
}

\begin{abstract}
The robust $L_{2}$-gain estimation is investigated for general uncertain systems with structured uncertainties. A new estimation structure is introduced: the Augmented-Gain Observer which encompasses both filters and observers and allows robust estimation even for some classes of unstable systems. Our approach is based on a separation of graphs theorem using frequency dependent Integral Quadratic Constraints. We prove that the design of an Augmented-Gain Observer ensuring a robust $L_{2}$-gain performance can be expressed as a convex optimization problem. This problem involves Linear Matrix Inequalities constraints and can be solved using an efficient algorithm. A numerical example illustrates the interest of the method.
\end{abstract}

\section{INTRODUCTION}

In many applications, it is often useful to measure some state vector components of a system for diagnosis, control, or supervision. If the necessary state vector components are not measured, they have to be estimated from the measure of other components of this vector, based on a model of the system. If this model is assumed to be linear time invariant and to perfectly represent the real system, computing an estimator has been largely investigated [8].

Two structures are mainly designed for the estimation. In this paper, these structures are referred to as the observer structure and the filter structure. An observer explicitly contains a model of the system whose dynamical evolution is driven through a gain by the error between the measured output and the estimated one [13]. A filter is defined as a (matrix of) transfer function(s) between the measured output and the estimation of the state components. Many approaches were proposed, for different puroposes: unknown initial conditions (Luenberger Observer [13]), stochastic perturbations (Kalman observer [10]), minimization of a norm of the estimation error $\left(H_{2}\right.$ or $H_{\infty}$ [23], [12]) The approaches proposed for the direct synthesis of a filter cannot be applied in the case of unstable systems.

These approaches do not take into account the modeling error, which can have a strong impact on the performance level achieved on the real system [2]. To deal with this difference the modeling error is represented with uncertainties [27]. The robust estimation problem is to find a robust estimator which ensures a guaranteed performance for all the systems represented by the uncertain model.

In the case of linear systems, two classes of uncertainties are in general represented: uncertainties on the model parameters (parametric uncertainties) and uncertainties on

\footnotetext{
${ }^{1}$ The authors are with Laboratoire Ampère, Ecole Centrale de Lyon, Université de Lyon, 36 avenue Guy de Collongue, 69134 Ecully Cedex, France. For correspondance, adress to benoit.bayon@ec-lyon.fr, gerard.scorletti@ec-lyon.fr, eric.blanco@ec-lyon.fr
}

the model dynamics (dynamical uncertainties). Results were proposed for a non structured parametric uncertainty: the state space matrices are assumed to be affine [5], [18] or rational [12], [3] functions of a unique uncertain matrix. Unfortunately, in general, there are several (scalar) parameter uncertainties in a model. A first simple case is when the state space matrices are affine in the uncertain parameters. In this case, referred to as polytopic systems [9], [26], [1], [7], [11], [17], the matrices of state-space representations lie in polytopes.

A more general interesting case is the case when matrices of state-space representations are rational functions of several parametric or dynamic uncertainties [25]. These systems can be represented as Linear Fractional Transformations (LFT) of a structured uncertainty $\Delta$ block by a nominal system $G$ [27]. The advantageous feature is that general linear systems with both parameter and dynamical uncertainties (including e.g. delays) can be represented as LFT [22], [21], [3]. This representation is actually one of the most general kind of representation [27].

In the LFT framework, the uncertain block is related to the nominal system using Integral Quadratic Constraints (IQCs) [14]. These IQCs allow to characterize a system using its input signals and its output signals. These IQCs provide us general analysis tools for the uncertain systems, such as stability tools, or performance analysis tools, using a Separation of Graph argument [20]. In [12], [25], the $H_{2}$ and $H_{\infty}$ filtering problems are considered for parametric time-varying uncertainties. The proposed approach can be interpreted in terms of static (constant) IQCs. In [22], [21], the filtering problem is tackled using dynamic (frequencydependent) IQCs in order to reduce the conservatism for time invariant parametric uncertainties with respect to the use of static IQCs. The problem is recast as an optimization problem involving LMIs using the Kalman-YakubovitchPopov Lemma [19]. The discrete time $H_{2}$ case has been solved with FIR filters [3]. In addition to be a more general approach of the robust filtering (due to rational parametric uncertainty dependence and the dynamical uncertainties), less conservative conditions are possibly obtained in the case of time-invariant uncertainties.

The major issue with all the previous approaches to the robust estimation problem is that the designed estimator is a filter. The proposed approach cannot be applied in the case of unstable systems. In some cases, this assumption is not satisfied as in [24]: it is then necessary to design an observer.

In this paper, we propose a new method for designing an $L_{2}$-gain sub-optimal observer in the case of uncertain systems in the LFT framework using the IQC/Separation of 
Graph approach. The proposed results pertain to our longstanding effort for investigating the robust filtering problem: in [16] with the desensitivity problem, in [22], [3] where this approach is applied to the design of some robust filters and in [15] where the benefits of new observer structures were emphasized. To our best knowledge, results on robust estimation using observers for such general uncertain models do not exist.

To this purpose, we propose a new structure for robust estimation with structured uncertainties. This new structure, referred to as the Augmented-Gain Observer (AGO), can be interpreted as the combination of an observer structure and a filter one. It contains the nominal model of the system, and a frequency-dependent gain to tackle the sensitivity problem. With this structure, it is then possible to make robust estimation for some classes of unstable systems.

The structured representation of uncertainties is very general and we use dynamics IQCs used only in [22], [21] to reduce the conservatism. Restricting ourselves to parametric/dynamic Linear Time Variant/Invariant uncertainties, we present a theorem to synthesize a $L_{2}$-gain suboptimal Augmented-Gain Observer as a finite dimensional, convex optimization problem. Nevertheless, the result can be obtained for more general uncertainties (such as nonlinear ones).

\section{Notations}

$\|\Delta\|_{i_{2}}$ is the induced norm by the $L_{2}$ space of signals. $A^{*}$ denotes the transpose conjugate of the complex matrix function $A . A^{T}$ is its transpose. The $\star$ denotes the Redheffer star product. $T_{w \rightarrow e}$ denotes the operator from $w$ to $e$. The Kronecker product is noted $\otimes$. The identity matrix is written $I$. The dimensions are appropriate in the equations. $G=$ $\left[\begin{array}{c|c}A & B \\ \hline C & D\end{array}\right]$ denotes the state space representation of $G(s)=$ $C(s I-A)^{-1} B+D$. Finally, $\operatorname{diag}(A, B)$ is a diagonal block matrix $\left[\begin{array}{c|c}A & 0 \\ \hline 0 & B\end{array}\right]$. $\bullet$ is a term of no importance, and $\diamond$ denotes a symmetric element in a symmetric matrix. We also define the following expression $W(A, B, C, D, P, X, T)$ $=\left[\begin{array}{c|c}A^{T} P+P A & P B \\ \hline B^{T} P & T\end{array}\right]+\left[\begin{array}{c}C^{T} \\ D^{T}\end{array}\right] X\left[\begin{array}{c}C^{T} \\ D^{T}\end{array}\right]^{T}$.

\section{The Robust $L_{2}$-Gain ObServation Problem}

\section{A. Problem Definition}

The uncertainty set is defined by :

$$
\Gamma_{\Delta}=\left\{\Delta \mid\left\{\begin{array}{l}
\|\Delta\|_{i_{2}}<1 \\
\Delta=\operatorname{diag}\left(\operatorname{diag}\left(\delta_{i}^{T V}\right), \operatorname{diag}\left(\Delta_{j}^{T V}\right), \ldots\right. \\
\left.\ldots, \operatorname{diag}\left(\delta_{k}^{T I}\right), \operatorname{diag}\left(\Delta_{l}^{T I}\right)\right)
\end{array}\right\}\right.
$$

- $\delta_{i}^{T V}$ is a time-variant real scalar

- $\Delta_{j}^{T V}$ is a time-variant Multi-Inputs Multi-Outputs (MIMO) operator

- $\delta_{k}^{T I}$ is a time-invariant real scalar

- $\Delta_{l}^{T I}$ is a time-invariant MIMO transfer function

The structure of the problem is presented in figure 1 . The plant $G$ is interconnected with the $\Delta$ operator. The output to

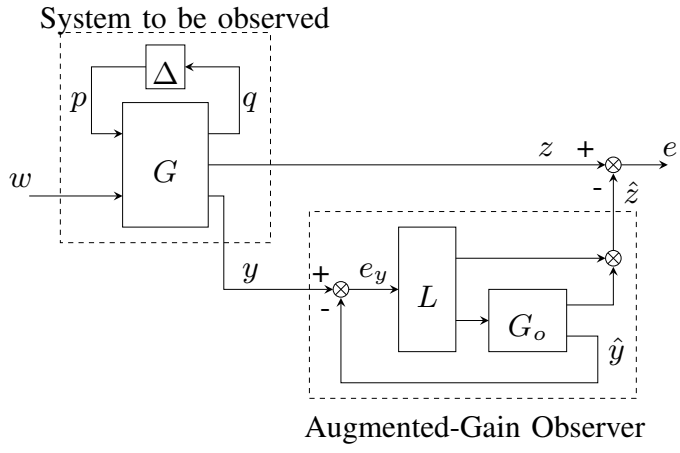

Fig. 1. The robust observation problem

be estimated is $z \in \mathbb{R}^{n_{z}}$, the measured output is $y \in \mathbb{R}^{n_{y}}$ and $p \in \mathbb{R}^{n_{p}}$ and $q \in \mathbb{R}^{n_{q}}$ are respectively the outputs and the inputs of the uncertainty bloc. The state space representation of the plant is:

$$
G=\left[\begin{array}{c|cc}
\tilde{A} & \tilde{B}_{p} & \tilde{B}_{w} \\
\hline \tilde{C}_{q} & \tilde{D}_{q p} & \tilde{D}_{q w} \\
\tilde{C}_{z} & \tilde{D}_{z p} & \tilde{D}_{z w} \\
\tilde{C}_{y} & \tilde{D}_{y p} & \tilde{D}_{y w}
\end{array}\right] .
$$

The purpose of the observer is to estimate the output $z$ from the measure of the input $y$. The minimal representation of the nominal system (for $\Delta=0$ ) is $G_{n}=\left[\begin{array}{c|c}A & B_{w} \\ \hline C_{z} & D_{z w} \\ C_{y} & D_{y w}\end{array}\right]$. The state-space representations of the estimator blocks are given by $G_{o}=\left[\begin{array}{c|c}A & I \\ \hline C_{z} & 0 \\ C_{y} & 0\end{array}\right]$ and $L=\left[\begin{array}{c|c}A_{L} & B_{L} \\ \hline C_{d} & D_{d} \\ C_{L} & D_{L}\end{array}\right]$.

The observer contains the nominal dynamics $G_{O}$ of the plant and a comparison between the measured output and its estimation. This innovation term $e_{y}$ is transmitted through the Augmented-Gain $L$. The original structure of Kalman [10] or Luenberger [13] can be retrieved by choosing $A_{L}, B_{L}$, $C_{L}, C_{d}, D_{d}$ as empty matrices. The filter structure can be recovered by choosing $C_{L}, D_{L}$ as zeros matrices and by withdrawing $G_{o}$ the central model from the estimator and the corresponding output of the augmented gain [22].

The $L_{2}$-gain observation problem is defined as follows: For a given $\gamma>0$, find if there exist an augmented gain $L$ such that

$$
\forall \Delta \in \Gamma_{\Delta}, \quad\left\|T_{w \rightarrow e}\right\|_{i_{2}}<\gamma
$$

and compute it.

\section{B. About the Augmented-Gain Observer}

The choice of the estimation structure is developed in this part, to present how the AGO allows to take into account some classes of uncertain systems. Basically, the observer structure allows us to work on a minimal system for a observation problem. The states of this system represent the error between the states of the nominal plant and the states simulated in the observer. This form is obtained using the following lemma: 
Lemma 1 (System reduction Lemma for observers):

Given $G$ a matrix of transfer functions defined by the following state space representation:

$$
G=\left[\begin{array}{cccc|c}
A_{1} & 0 & 0 & 0 & B_{1} \\
0 & A_{2} & 0 & 0 & B_{2} \\
0 & C_{2} & A_{2}-C_{2} & C_{3} & B_{3} \\
0 & C_{4} & -C_{4} & A_{3} & B_{4} \\
\hline C_{1} & 0 & 0 & 0 & D_{1} \\
0 & C_{5} & -C_{5} & C_{6} & D_{2}
\end{array}\right] .
$$

Then $G$ also admits the following state space representation:

$$
G_{\min }=\left[\begin{array}{ccc|c}
A_{1} & 0 & 0 & B_{1} \\
0 & A_{2}-C_{2} & C_{3} & B_{2}-B_{3} \\
0 & C_{4} & A_{3} & B_{4} \\
\hline C_{1} & 0 & 0 & D_{1} \\
0 & C_{5} & C_{6} & D_{2}
\end{array}\right] .
$$

Proof: The state-space transformation $T$ is used:

$$
T=\operatorname{diag}\left(I,\left[\begin{array}{cc}
I & 0 \\
I & -I
\end{array}\right], I\right) \text {. }
$$

The state space representation obtained is a Kalman form of observability, and can therefore be reduced from an observability point of view. The complete proof can be found in [13].

This lemma is applied on the robust observation problem (figure 1). The plant $G$ defined equation (2) is separated in two minimal parts $H$ and $G_{N}$,

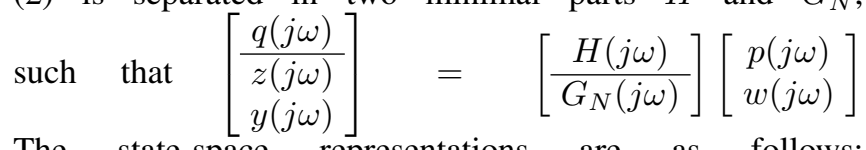
The state-space representations are as follows:

$$
H=\left[\begin{array}{c|cc}
A_{H} & B_{H p} & B_{H w} \\
\hline C_{H} & D_{H p} & D_{H w}
\end{array}\right] \quad G_{N}=\left[\begin{array}{c|cc}
A & B_{p} & B_{w} \\
\hline C_{z} & D_{z p} & D_{z w} \\
C_{y} & D_{y p} & D_{y w}
\end{array}\right]
$$

$H$ is the part that acts on the uncertainty bloc. When $H=0$ or $\Delta=0$ there is no uncertainty on the model. $G_{N}$ represents the nominal model augmented with the uncertainty entry $p$. This case recovers the nominal case, without uncertainties. A complete representation $G_{t o t}$ of the system withdrawing the uncertain block is:

$$
\begin{aligned}
& {\left[\begin{array}{cccc|c}
A_{H} & 0 & 0 & 0 & B_{H} \\
0 & A & 0 & 0 & B_{G} \\
0 & D_{L} C_{y} & A-D_{L} C_{y} & C_{L} & D_{L} D_{y} \\
0 & B_{L} C_{y} & -B_{L} C_{y} & A_{L} & B_{L} D_{y} \\
\hline C_{H} & 0 & 0 & 0 & D_{H} \\
0 & C_{z}-D_{d} C_{y} & -C_{z}+D_{d} C_{y} & -C_{d} & D_{z}-D_{d} D_{y}
\end{array}\right]} \\
& \left.\begin{array}{c}
B_{H} \\
B_{G} \\
D_{L} D_{y} \\
B_{L} D_{y} \\
\hline D_{H} \\
D_{z}-D_{d} D_{y}
\end{array}\right]=\left[\begin{array}{cc}
B_{H p} & B_{H w} \\
B_{p} & B_{w} \\
D_{L} D_{y p} & D_{L} D_{y w} \\
B_{L} D_{y p} & B_{L} D_{y w} \\
\hline D_{H p} & D_{H w} \\
D_{z p}-D_{d} D_{y p} & D_{z w}-D_{d} D_{y w}
\end{array}\right]
\end{aligned}
$$

Applying lemma 1, a minimal state space representation of $G_{r}$ is:

$$
\left[\begin{array}{c}
H(j \omega) \\
G_{m}(j \omega)
\end{array}\right]=\left[\begin{array}{ccc|c}
A_{H} & 0 & 0 & B_{H} \\
0 & A-D_{L} C_{y} & C_{L} & B_{G}-D_{L} D_{y} \\
0 & B_{L} C_{y} & A_{L} & B_{L} D_{y} \\
\hline C_{H} & 0 & 0 & D_{H} \\
0 & C_{z}-D_{d} C_{y} & -C_{d} & D_{z}-D_{d} D_{y}
\end{array}\right]
$$

This minimal system is used to compute the augmented-gain.

\section{UNCERTAIN SYSTEMS ANALYSIS}

In order to synthesize the observer gain, analysis tools for the uncertain systems are needed. Those are given by the IQCs framework which allows to characterize a system through constraints over its inputs and its outputs. The constraints for LTV and LTI uncertainties are now presented. Associated to the set of uncertainties, the set of hermitian bounded matrices of transfer functions $\bar{\Pi}\left(\Gamma_{\Delta}\right)$ is presented.

$$
\Pi(j \omega)=\left[\begin{array}{cc}
\Pi_{s}(j \omega) & \Pi_{a s}(j \omega) \\
\Pi_{a s}(j \omega)^{*} & -\Pi_{s}(j \omega)
\end{array}\right]
$$

with the following definitions:

$\Pi_{s}(j \omega)=\operatorname{diag}\left(\operatorname{diag}\left(S_{i}\right), \operatorname{diag}\left(d_{j}\right), \operatorname{diag}\left(S_{k}(j \omega)\right) \operatorname{diag}\left(s_{l}(j \omega)\right)\right)$ $\Pi_{a s}(j \omega)=\operatorname{diag}\left(\operatorname{diag}\left(Y_{i}\right), 0, \operatorname{diag}\left[Y_{k}(j \omega)\right], 0\right)$ where $S_{i}=$ $S_{i}^{T}>0, Y_{i}=-Y_{i}^{T}, d_{j}>0, S_{k}(j \omega)=S_{k}(j \omega)^{*}>0$, $Y_{k}(j \omega)=-Y_{k}(j \omega)^{*}, s_{l}(j \omega)=s_{l}(j \omega)^{*}>0$.

From [14], we get the following analysis result:

Theorem 2: Robust $L_{2}$-Gain analysis

Let $M(j \omega)$ be LTI, for all $\Delta \in \Gamma_{\Delta}, \Delta \star M$ is stable with an $L_{2}$-Gain less than $\gamma$ if there exists $\Pi \in \bar{\Pi}\left(\Gamma_{\Delta}\right)$ and $\epsilon>0$ such that for all $\omega$ :

$$
\begin{gathered}
{\left[\frac{M(j \omega)}{I}\right]^{*} \Phi\left[\frac{M(j \omega)}{I}\right] \leq-\epsilon I} \\
\Phi=\left[\begin{array}{cc|cc}
\Pi_{s}(j \omega) & 0 & \Pi_{a s}(j \omega) & 0 \\
0 & I & 0 & 0 \\
\hline \Pi_{a s}^{*}(j \omega) & 0 & -\Pi_{s}(j \omega) & 0 \\
0 & 0 & 0 & -\gamma^{2} I
\end{array}\right]
\end{gathered}
$$

For a given system and a given $\gamma$, to find $\Pi(j \omega)$ such that the condition (5) is satisfied is an optimization problem. But it is an infinite dimensional one, as all the possible transfer functions of every order are candidates. In order to get a finite-dimension optimization problem, the matrix of transfer function $\Pi(j \omega)$ is restricted to rational transfer function matrices with a fixed order in numerator and a fixed denominator $f(j \omega)$, a Hurwitz polynomial.

$$
\Pi(j \omega)=\operatorname{diag}(K(j \omega), K(j \omega))^{*} X \operatorname{diag}(K(j \omega), K(j \omega))
$$

The quadratic condition (5) can be recast as

$$
\left[\begin{array}{cc|cc}
K^{*}(j \omega) & 0 & 0 & 0 \\
0 & I & 0 & 0 \\
\hline 0 & 0 & K^{*}(j \omega) & 0 \\
0 & 0 & 0 & I
\end{array}\right] \Phi_{X}\left[\begin{array}{cc|cc}
K(j \omega) & 0 & 0 & 0 \\
0 & I & 0 & 0 \\
\hline 0 & 0 & K(j \omega) & 0 \\
0 & 0 & 0 & I
\end{array}\right] .
$$

with

$$
\begin{aligned}
& K(j \omega)=\operatorname{diag}\left(\operatorname{diag}\left(I_{i}\right), \operatorname{diag}\left(I_{j}\right), \ldots\right. \\
& \left.\ldots \operatorname{diag}\left(\frac{E(j \omega)}{f(j \omega)} \otimes I_{k}\right), \operatorname{diag}\left(\frac{E(j \omega)}{f(j \omega)} \otimes I_{l}\right)\right)
\end{aligned}
$$

defined as :

$$
\Phi_{X}=\left[\begin{array}{cc|cc}
X_{s} & 0 & X_{a s} & 0 \\
0 & I & 0 & 0 \\
\hline X_{a s}^{T} & 0 & -X_{s} & 0 \\
0 & 0 & 0 & -\gamma^{2} I
\end{array}\right] .
$$

$E(j \omega)$ is a basis for the possible transfer functions:

$$
E(j \omega)=\left[\begin{array}{llll}
(j \omega)^{n} & (j \omega)^{n-1} & \ldots & 1
\end{array}\right]^{T} .
$$


We define the set of matrices $X \in \bar{X}(\bar{\Pi})$, which generates the transfer functions

$$
\begin{aligned}
\text { - } & X= \\
\text { - } X_{s}= & {\left[\begin{array}{cc}
X_{s} & X_{a s} \\
X_{a s}^{T} & -X_{s}
\end{array}\right] } \\
& \quad \ldots, \operatorname{diag}\left[\operatorname{diag}\left(S_{i}\right), \operatorname{diag}\left(X_{k}^{S}\right), \operatorname{diag}\left(I \otimes x_{l}\right)\right] \\
\text { - } X_{a s}= & \operatorname{diag}\left[\operatorname{diag}\left(G_{i}\right), 0_{j}, \operatorname{diag}\left(X_{k}^{G}\right), 0\right]
\end{aligned}
$$

With this representation, the problem has a semi-finite dimension: the decision variables are no longer the $\Pi(j \omega)$ transfer functions, but the finite-dimensional matrices $X_{k}^{S}$ and $X_{k}^{G}$. The number of constraints is still infinite as all the frequencies have to be tested. For the particular structure of $X_{k}^{S}$ and $X_{k}^{G}$, an economical parametrization can be chosen, see [22].

As the quadratic condition is now defined, the analysis problem can be recast as a finite dimensional optimization problem under LMIs constraints using the KalmanYakubovitch-Popov Lemma [19].

In this part the fundamental analysis tools for uncertain systems have been presented with the steps to transform the analysis condition in a convex finite-dimensional optimization problem.

\section{MAIN RESULT}

In this section, we present the theorem which allows to test the existence of an Augmented-Gain Observer (AGO) solution of the $L_{2}$-gain observation problem presented section II-A. The proof is develloped using the tools presented in section II-B and section III.

Theorem 3: For a given $\gamma>0$, the robust observation problem has a solution if there exists $P_{K}=P_{K}^{T}, R=R^{T}$, $Z=Z^{T}>0, X \in \bar{X}$ of appropriate dimensions such that the following conditions are satisfied.

1) $R-\operatorname{diag}(Z, 0)>0$

2)

$$
U_{R \perp}^{T}\left[\begin{array}{c|c}
W\left(A_{R}, B_{R}, C_{\Delta}, \ldots\right. & {\left[\begin{array}{c}
C_{R}^{T} \\
D_{R}^{T}
\end{array}\right]} \\
\left.\hline \ldots, D_{\Delta}, R, X, T(\gamma)\right) & -I
\end{array}\right] U_{R \perp}<0
$$$$
\text { with } U_{R \perp}^{T}=\operatorname{diag}\left(I, I, I,\left[\begin{array}{lll}
N_{1}^{T} & N_{2}^{T} & N_{3}^{T}
\end{array}\right], I\right) \text {. }
$$

$$
\left[\begin{array}{lll}
N_{1}^{T} & N_{2}^{T} & N_{3}^{T}
\end{array}\right]=\left[\begin{array}{lll}
C_{y} & D_{y p} & D_{y}
\end{array}\right]^{\perp}
$$

and $T(\gamma)=\operatorname{diag}\left(0,-\gamma^{2} I\right)$

3) $W\left(A_{Z}, B_{Z}, C_{\Delta Z}, D_{\Delta}, Z, X, T(\gamma)\right)<0$

4) $\left.W\left(A_{K}, B_{K}, C_{K}, D_{K}, P_{K}, X_{s}, 0\right)\right)>0$

with the following matrices:

$$
\begin{aligned}
& {\left[\begin{array}{c|c}
A_{Z} & B_{Z} \\
\hline C_{\Delta_{Z}} & D_{\Delta}
\end{array}\right]=\left[\begin{array}{ccc|cc}
A_{H} & 0 & 0 & B_{H p} & B_{H w} \\
B_{K} C_{H} & A_{K} & 0 & B_{K} D_{H p} & B_{K} D_{H w} \\
0 & 0 & A_{K} & B_{K} & 0 \\
\hline D_{K} C_{H} & C_{K} & 0 & D_{K} D_{H p} & D_{K} D_{H w} \\
0 & 0 & C_{K} & D_{K} & 0
\end{array}\right]} \\
& {\left[\begin{array}{c|c}
A_{R} & B_{R} \\
\hline C_{\Delta} & D_{\Delta} \\
\hline C_{R} & D_{R}
\end{array}\right]=\left[\begin{array}{cc|c}
A_{Z} & 0 & B_{Z} \\
0 & A & B_{p} \\
\hline C_{\Delta_{Z}} & 0 & B_{w} \\
\hline 0 & C_{z} & D_{z p} \\
\hline & D_{z w}
\end{array}\right]}
\end{aligned}
$$

$K=\left[\begin{array}{l|l}A_{K} & B_{K} \\ \hline C_{K} & D_{K}\end{array}\right]$ is the state space representation used for the IQC factorization presented equation (7).
These 4 conditions define a convex feasibility problem under LMI constraints. If they are satisfied, an observer with an Augmented-Gain exists such that the $L_{2}$ gain of the estimation error is less than $\gamma$. Minimizing over $\gamma$ under these conditions gives an upper bound on the best performance reachable for the estimation error. Basically, the second condition can be interpreted as a generalization of the observability condition on the system. The third condition of the theorem ensures the stability of the block $H \star \Delta$ which is necessary for the whole system to be stable. This can be proven by applying the KYP lemma on this condition. The first condition with $Z>0$ ensures that the $P$ matrix introduced with the KYP lemma is defined positive, which is a condition for the system to be stable [6]. The augmented gain has yet to be reconstructed.

Proof: Applying theorem 2 to system defined equation (4), we have:

$$
\left[\begin{array}{c}
H(j \omega) \\
\frac{\left[\begin{array}{ll}
I & 0
\end{array}\right]}{G_{m}(j \omega)} \\
{\left[\begin{array}{cc}
0 & I
\end{array}\right]}
\end{array}\right]^{*}\left[\begin{array}{ccc}
\Pi(j \omega) & 0 & 0 \\
\hline 0 & I & 0 \\
0 & 0 & -\gamma^{2} I
\end{array}\right]\left[\begin{array}{c}
H(j \omega) \\
\frac{\left[\begin{array}{ll}
I & 0
\end{array}\right]}{G_{m}(j \omega)} \\
{\left[\begin{array}{ll}
0 & I
\end{array}\right]}
\end{array}\right]<-\epsilon I
$$

This constraint can be recast with the factorization presented section III, equation (6) as:

$$
\left[\begin{array}{c}
K(j \omega) H(j \omega) \\
{\left[\begin{array}{cc}
K(j \omega) & 0
\end{array}\right]} \\
\hline G_{m}(j \omega) \\
{\left[\begin{array}{ll}
0 & I
\end{array}\right]}
\end{array}\right]^{*}\left[\begin{array}{c|cc}
X & 0 & 0 \\
\hline 0 & I & 0 \\
0 & 0 & -\gamma^{2} I
\end{array}\right]\left[\begin{array}{c}
K(j \omega) H(j \omega) \\
{\left[\begin{array}{c}
K(j \omega) \\
G_{m}(j \omega)
\end{array}\right]} \\
{\left[\begin{array}{ll}
0 & I
\end{array}\right]}
\end{array}\right]<-\epsilon I
$$

The state space representation of the system is:

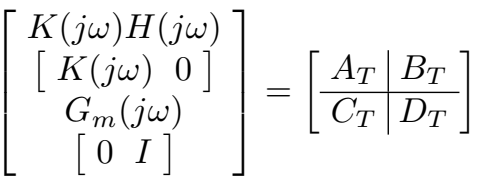

$$
\begin{gathered}
{\left[\begin{array}{c}
A_{T} \\
\hline C_{T}
\end{array}\right]=\left[\begin{array}{ccccc}
A_{H} & 0 & 0 & 0 & 0 \\
B_{K} C_{H} & A_{K} & 0 & 0 & 0 \\
0 & 0 & A_{K} & 0 & 0 \\
0 & 0 & 0 & A-D_{L} C_{y} & C_{L} \\
0 & 0 & 0 & B_{L} C_{y} & A_{L} \\
\hline D_{K} C_{H} & C_{K} & 0 & 0 & 0 \\
0 & 0 & C_{K} & 0 & 0 \\
0 & 0 & 0 & C_{z}-D d C_{y} & -C d \\
0 & 0 & 0 & 0 & 0
\end{array}\right]} \\
{\left[\begin{array}{c}
B_{T} \\
\hline D_{T}
\end{array}\right]=\left[\begin{array}{cc}
B_{H p} & B_{H w} \\
B_{K} D_{H p} & B_{K} D_{H w} \\
B_{K} & 0 \\
B_{p}-D_{L} D_{y p} & B_{w}-D_{L} D_{y w} \\
B_{L} D_{y p} & B_{L} D_{y w} \\
\hline D_{K} D_{H p} & D_{K} D_{H w} \\
D_{K} & 0 \\
D_{z p}-D_{d} D_{y p} & D_{z w}-D_{d} D_{y} \\
0 & I
\end{array}\right]}
\end{gathered}
$$

Using the KYP lemma [19], the condition (11) is equivalent to the existence of $P=P^{T}>0$ such that the condition $W\left(A_{T}, B_{T}, C_{T}, D_{T}, P, \Phi_{T}, 0\right)<0$ holds, with

$$
\Phi_{T}=\operatorname{diag}\left(\left[\begin{array}{cc}
X_{s} & X_{a s} \\
X_{a s}^{T} & -X_{s}
\end{array}\right], I,-\gamma^{2} I\right)
$$


Using a Schur complement ([4, page 7$])$, we have: $\left[\begin{array}{c|c}W\left(A_{T}, B_{T},\left[\begin{array}{c}C_{\Delta} \\ 0\end{array}\right], \ldots\right. & {\left[\begin{array}{c}\left(C_{R}-D_{d} \bar{C}_{y}\right)^{T} \\ \left.\ldots, D_{\Delta}, P, X, T(\gamma)\right)\end{array}\right.} \\ \hline \diamond & {\left[\begin{array}{c}T \\ \left(D_{z p}-D_{d} D_{y p}\right)^{T} \\ \left(D_{z w}-D_{d} D_{y}\right)^{T}\end{array}\right]}\end{array}\right]<0$

This condition is a Bilinear Matrix Inequality, and has to be linearized. It could be written as:

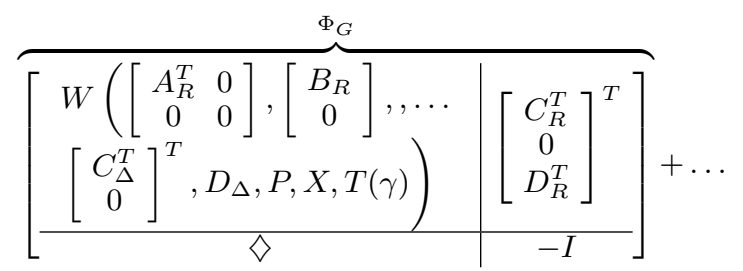

$$
\begin{gathered}
+V \overbrace{\left[\begin{array}{l|l}
A_{L} & B_{L} \\
\hline C_{L} & D_{L} \\
C_{d} & D_{d}
\end{array}\right]}^{\Phi_{L}} \overbrace{\left[\begin{array}{lll}
U_{1} & U_{2} & 0
\end{array}\right]}^{U}+\ldots \\
\cdots+U^{T} \Phi_{L}^{T} \overbrace{\left[\begin{array}{lll}
V_{1} P & 0 & V_{2}
\end{array}\right]}^{V}<0
\end{gathered}
$$

with $\left[V_{1} \mid V_{2}\right]=\left[\begin{array}{ccccc|c}0 & 0 & 0 & 0 & I & 0 \\ 0 & 0 & 0 & -I & 0 & 0 \\ 0 & 0 & 0 & 0 & 0 & -I\end{array}\right]$ and

$$
\left[U_{1} \mid U_{2}\right]=\left[\begin{array}{ccccc|cc}
0 & 0 & 0 & 0 & I & 0 & 0 \\
0 & 0 & 0 & C_{y} & 0 & D_{y p} & D_{y}
\end{array}\right] \text {. }
$$

We can apply the elimination lemma [4, page 22]. The basis of the nullspaces of the matrices $V$ and $U$ presented equation (13) are:

$$
\begin{aligned}
& \text { - } V_{\perp}=\operatorname{diag}\left(P^{-1}, I, I\right)\left[\begin{array}{cccc}
\operatorname{diag}(I, I, I, 0) \\
\hline 0 & 0 & 0 & I \\
\hline 0 & 0 & 0 & 0
\end{array}\right] \\
& \text { - } U_{\perp}^{T}=\operatorname{diag}\left(I, I, I,\left[\begin{array}{llll}
N_{1}^{T} & 0 & N_{2}^{T} & N_{3}^{T}
\end{array}\right], I\right)
\end{aligned}
$$

The partitions of the $\mathrm{P}$ matrix and $P^{-1}$ are introduced.

$$
P=\left[\begin{array}{cc}
R & \bullet \\
\bullet & \bullet
\end{array}\right], P^{-1}=\left[\begin{array}{cc}
\hat{R} & \bullet \\
\bullet & \bullet
\end{array}\right], \hat{R}=\left[\begin{array}{cc}
Z^{-1} & \hat{R}_{2} \\
\hat{R}_{2}^{T} & \hat{R}_{3}
\end{array}\right]
$$

The first constraint $U_{\perp}^{T} \Phi_{G} U_{\perp}<0$ gives by applying the elimination lemma and due of the line of zeros in $U_{\perp}$ the second condition of the theorem.

The second constraint $V_{\perp}^{T} \Phi_{G} V_{\perp}<0$ could be simplified due to the zeros on the external terms to:

$$
W\left(A_{Z} Z^{-1}, B_{Z}, Z^{-1} C_{\Delta Z}, D_{\Delta}, I, X, T(\gamma)\right)<0
$$

Then, using a congruent multiplication by $J=\operatorname{diag}(Z, I)$ we get the third condition of the theorem.

Finally, the use of the elimination lemma introduces a rank constraint over the partitions of the Lyapunov matrix and its inverse [6]:

$$
\left[\begin{array}{c|cc}
R & \multicolumn{1}{|c}{I} \\
\hline I & Z^{-1} & \hat{R}_{2} \\
& \hat{R}_{2}^{T} & \hat{R}_{3}
\end{array}\right]>0
$$

but as the partition $\hat{R}_{2}$ and $\hat{R}_{3}$ only appears in this condition, $\hat{R}_{3}$ can be eliminated using the elimination lemma once again, and then a Schur complement give us the first condition of theorem $R-\operatorname{diag}(Z, 0)>0$.

The transfer function $\Pi_{s}$ is defined positive:

$$
K(j \omega)^{*} X_{s} K(j \omega)>\epsilon I
$$

The application of the KYP lemma gives the fourth condition [19].

\section{OBSERVER RECONSTRUCTION}

Reconstructing the augmented-gain of the observer from the equation (13) is an optimization problem under LMI constraints. But the original $P$ matrix introduced by the KYP lemma of this problem has to be reconstructed first. As $Z^{-1}$ is a partition of $\hat{R}$, itself a partition of $P^{-1}$, find $\hat{R}$ is a feasibility problem under LMI constraints, and the constraint is given by the equation (14). $\mathrm{P}$ is obtained using the following parametrization:

$$
P=\left[\begin{array}{cc}
I & 0 \\
0 & M^{T}
\end{array}\right]\left[\begin{array}{cc}
R & I \\
I & \left(R-\hat{R}^{-1}\right)^{-1}
\end{array}\right]\left[\begin{array}{cc}
I & 0 \\
0 & M
\end{array}\right]
$$

Once $P$ is obtained, to find the state-space representation of the augmented gain can be done solving an optimization problem given by the constraint (13). As $P, X$ are known, this constraint is an LMI constraint and the reconstruction can be made as a feasibility problem.

\section{EXAMPLE}

In this section, we present two numerical applications of the AGO. We first compare it with the other methods using dynamic IQCs, on an example of the literature. We then present an example of random walk [24], where the previous methods do not lead to feasible conditions.

We first consider the application presented in [21], for the synthesis of an $L_{2}$-Gain estimator. We compare the methods presented in [22], [21], which gives equivalent results with different conditions, and our method, with the same basis for the dynamic IQCs. The denominator is chosen as $f=$ $(s+0.01)^{n}$, with $\mathrm{n}$ varying from 0 (static IQCs) to 5 . The results are presented table I. The results proves that the AGO, recovers the methods presented in the same framework.

\begin{tabular}{|c|c|c|c|c|}
\hline Method & $n=0$ & $n=1$ & $n=3$ & $n=5$ \\
\hline$[22],[21]$ & 4.54 & 3.08 & 2.64 & 2.64 \\
\hline AGO & 4.54 & 3.08 & 2.64 & 2.64 \\
\hline
\end{tabular}

TABLE I

COMPARISON OF METHODS

We present an example of uncertain random walk. The system is defined ad follows:

$$
\left[\begin{array}{l}
z \\
y
\end{array}\right]=\left[\begin{array}{c}
\left(\frac{a_{\delta}}{s^{2}+b a_{\delta} s+a_{\delta}}\right) \\
\left(\frac{1}{s}\right)\left(\frac{a_{\delta}}{s^{2}+b a_{\delta} s+a_{\delta}}\right)
\end{array}\right] w .
$$

We want to estimate $z$, from the output $y$. This is a first differentiation of $y$. A white noise $v(t)$ is added on the output, with a factor 0.1 . The second order transfer function is dependent from the parameter $a_{\delta}$ which nominal value $a_{n}$ is set to 1 . This parameter present a variation of $a_{g a b} \%$. This variation acts on the natural frequency of the second order filter. Note that as the systems contains a non-strictly stable pole, methods for synthesis of robust filters do not lead to feasible conditions. 
The state space representation of the uncertain random walk, as an LFT representation is presented equation (15).

$$
\left[\begin{array}{c}
\dot{x}_{1}(t) \\
\dot{x}_{2}(t) \\
\dot{x}_{3}(t) \\
\hline q(t) \\
\hline z(t) \\
\hline y(t)
\end{array}\right]=\left[\begin{array}{ccc|ccc}
-b a_{n} & -1 & 0 & -b & 1 & 0 \\
a_{n} & 0 & 0 & 1 & 0 & 0 \\
0 & 1 & 0 & 0 & 0 & 0 \\
\hline a_{g a b} & 0 & 0 & 0 & 0 & 0 \\
\hline 0 & 1 & 0 & 0 & 0 & 0 \\
\hline 0 & 0 & 1 & 0 & 0 & d
\end{array}\right]\left[\begin{array}{c}
x_{1}(t) \\
x_{2}(t) \\
x_{3}(t) \\
\hline p(t) \\
w(t) \\
v(t)
\end{array}\right]
$$

This example is tractable with our method. In order to illustrate the interest of dynamic IQCs, we apply theorem 3 on this system with static and dynamic IQCs. For the dynamic IQCs, the denominator is chosen as $f(s)=(s+0.1)^{n}$, for $n=1,2,3$. We compare the upper-bounds on the $L_{2}$ gain performance reached with those four cases (static IQCs, dynamic IQCs with $n=1,2,3$ ) versus $a_{g a b}$.

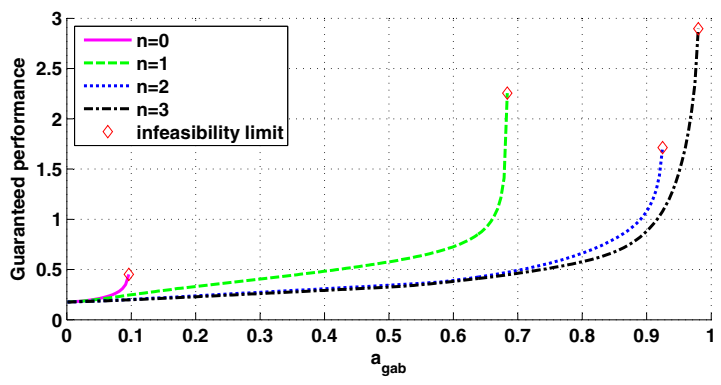

Fig. 2. Upper-bound on the performance versus $r_{g a b}$

Without uncertainties, we recover performance reached by the nominal $H_{\infty}$ observer. When the uncertainty grows, the constraints become infeasible depending on the conservatism of the method. The use of static IQC $(n=0)$ allows to take into account uncertainties on the parameter about $10 \%$. The use of dynamic IQCs allows to take into account uncertainties 9 times larger. These two examples prove that our observer gives the same performance as methods previously presented in this framework, but also allows to take into account cases non tractable with these methods.

\section{CONCLUSION}

In this paper, we present a new structure: the AugmentedGain Observer, which allows to observe uncertain systems such as systems with LTV/LTI uncertainties, or some unstable uncertain systems. This structure is very general, and encompasses most of the previous structures. We have presented the solution of the $L_{2}$-gain sub-optimal AugmentedGain Observer based on finite dimensional optimization, and a separation of graphs theorem. It has also been proved that the resolution presented could be used for other structures, such as static-gain observers. This solution is still conservative, but the conservatism could be reduced by increasing the order of the dynamic IQCs $\Pi(j \omega)$ at the cost of the number of variables. This resolution paves the way to a general approach of robust estimation for uncertain LFT systems. One of the closest perspectives is to compute an $H_{2}$ suboptimal augmented-gain observer.

\section{REFERENCES}

[1] K. Barbosa, C. De Souza, and A. Trofino. Robust $H_{2}$ filtering for uncertain linear systems: LMI based methods with parametric Lyapunov functions. Systems and Control Letters, 54(3):251-262, 2005

[2] S. Bhattacharyya. The structure of robust observers. IEEE Transactions on Automatic Control, 21(4):581-588, 1976.

[3] X. Bombois, H. Hjalmarsson, and G. Scorletti. Identification for robust $\mathrm{H}_{2}$ deconvolution filtering. Automatica, 46(3):577-584, Mar. 2010.

[4] S. Boyd, L. El Ghaoui, E. Feron, and V. Balakrishnan. Linear matrix inequalities in system and control theory. Society for Industrial Mathematics, 1994

[5] M. Fu, C. De Souza, and L. Xie. $H_{\infty}$ estimation for uncertain systems. International Journal of Robust and Non-Linear Control, 2:87-105, 1992.

[6] P. Gahinet and P. Apkarian. A linear matrix inequality approach to $H_{\infty}$ control. International Journal of Robust and Non-Linear Control, 4(4):421-448, 1994.

[7] H. Gao, X. Meng, and T. Chen. A new design of robust $H_{2}$ filters for uncertain systems. Systems and Control Letters, 57(7):585-593, 2008.

[8] A. Gelb. Applied optimal estimation. Wiley New York, 1974.

[9] J. Geromel and M. de Oliveira. $H_{2}$ and $H_{\infty}$ robust filtering for convex bounded uncertain systems. IEEE Transactions on Automatic Control, 46:100-107, 2001.

[10] R. Kalman. A New Approach to Linear Filtering and Prediction Problems. Transactions of the ASME - Journal of Basic Engineering, 82(Series D):35-45, 1960.

[11] R. Korogui and J. Geromel. $H_{2}$ robust filter design with performance certificate via convex programming. Automatica, 44(4):937-948, 2008.

[12] H. Li and M. Fu. A Linear Matrix Inequality Approach to Robust $H_{\infty}$ Filtering. IEEE Transactions on Signal Processing, 45(9):23382350, 1997.

[13] D. Luenberger. An introduction to observers. IEEE Transactions on automatic control, 16(6):596-602, 1971.

[14] A. Megretski and A. Rantzer. System analysis via integral quadratic constraints. IEEE Transactions on Automatic Control, 42(6):819-830, 1997.

[15] P. Neveux and E. Blanco. Robust $H_{\infty}$ filtering by means of lead-lag controller. In Proc. 17th IFAC World Congress, 2008.

[16] P. Neveux, E. Blanco, and G. Thomas. Robust Filtering for Linear Time-Invariant Continuous Systems. IEEE Transactions on Signal Processing, 55(10):4752-4757, Oct. 2007.

[17] R. M. Palhares and P. L. D. Peres. Robust $H_{\infty}$ Filtering Design with Pole Placement Constraint via Linear Matrix Inequalities. Journal of Optimisation Theory and Applications, 102(2):239-261, 1999.

[18] I. Petersen and D. McFarlane. Optimal guaranteed cost control and filtering for uncertain linear systems. IEEE Transactions on Automatic Control, 39(9):1971-1977, 1994.

[19] A. Rantzer. On the Kalman-Yakubovich-Popov lemma. Systems \& Control Letters, 28:7-10, 1996.

[20] M. Safonov. Stability and Robustness of Multivariable Feedback Systems. MIT Press, 1980.

[21] C. Scherer and I. Kose. Robustness with dynamic IQCs: An exact state-space characterization of nominal stability with applications to robust estimation. Automatica, 44(7):1666-1675, July 2008.

[22] G. Scorletti and V. Fromion. Further results on the design of robust $H_{\infty}$ feedforward controllers and filters. In Proceedings of the 45th IEEE Conference on Decision and Control, pages 3560-3565, 2006.

[23] U. Shaked. $H_{\infty}$ minimum error state estimation oflinear stationary processes. IEEE Transactions on Automatic Control, 35(5):554-558, 1990

[24] R. Singer. Estimating optimal tracking filter performance for manned maneuvering targets. IEEE Transactions On Aerospace And Electronic Systems, 6(4):pp. 473-483, 1970.

[25] K. Sun and A. Packard. Robust $H_{2}$ and $H_{\infty}$ filters for uncertain LFT systems. IEEE Transactions on Automatic Control, 50(5):715720, 2005.

[26] H. Tuan, P. Apkarian, and T. Nguyen. Robust and reduced-order filtering: new LMI-based characterizations and methods. 49(12):29752984, 2001.

[27] K. Zhou, J. Doyle, and K. Glover. Robust and Optimal Control. Prentice Hall, 1995. 